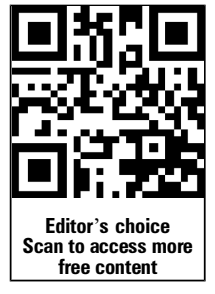

- Additional material is published online only. To view please visit the journal online (http://dx.doi.org/10.1136/ heartjnl-2014-305729).

${ }^{1}$ Institut Universitaire de Cardiologie et de Pneumologie de Québec/Québec Heart \& Lung Institute, Laval University, Québec city, Québec, Canada ${ }^{2}$ Department of Cardiology, GIGA Cardiovascular Sciences, University of Liège Hospital, Heart Valve Clinic, CHU Sart Tilman, Liège, Belgium

\section{Correspondence to} Dr Philippe Pibarot, Institut Universitaire de Cardiologie et de Pneumologie de Québec, 2725 Chemin Sainte-Foy, Québec city, Québec, Canada G1V-4G5;

philippe.pibarot@med.ulaval.ca

Professor Patrizio Lancellotti, CHU Start Tilman, Domaine Sart Tilman 1, Liège,

Belgium 4000;

plancellotti@chu.ulg.ac.be

$\mathrm{RC}$ and JM contributed equally to this study to this study.

Received 18 February 2014 Revised 29 May 2014

Accepted 1 June 2014

Published Online First

3 July 2014

\section{SLinked}

http://dx.doi.org/10.1136/ heartjnl-2014-306253

CrossMark

To cite: Capoulade $R$ Magne J, Dulgheru R, et al. Heart 2014;100

$1606-1612$.

\title{
Prognostic value of plasma B-type natriuretic peptide levels after exercise in patients with severe asymptomatic aortic stenosis
}

\author{
Romain Capoulade, ${ }^{1}$ Julien Magne, ${ }^{2}$ Raluca Dulgheru, ${ }^{2}$ Zeineb Hachicha, ${ }^{1}$ \\ Jean G Dumesnil, ${ }^{1}$ Kim O'Connor, ${ }^{1}$ Marie Arsenault, ${ }^{1}$ Sébastien Bergeron, ${ }^{1}$ \\ Luc A Pierard, ${ }^{2}$ Patrizio Lancellotti, ${ }^{2}$ Philippe Pibarot ${ }^{1}$
}

\begin{abstract}
Background Exercise-stress echocardiography is useful in management and risk stratification of patients with asymptomatic aortic stenosis (AS). Resting B-type natriuretic peptide (BNP) level is associated with increased risk of adverse events. The incremental prognostic value of BNP response during exercise is unknown.
\end{abstract}

Objective The purpose of this study was to assess the usefulness of plasma level of BNP during exercise to predict occurrence of events in asymptomatic patients with severe AS.

Methods Resting and exercise-stress echocardiographic data and plasma BNP levels were prospectively collected in 211 asymptomatic AS patients in whom 157 had severe AS with preserved LVEF in two centres. The study end-point was the occurrence of death or aortic valve replacement.

Results Plasma BNP level increased from rest to exercise $(p<0.0001)$. During a mean follow-up of 1.5 \pm 1.2 years, 87 patients with severe AS reached the predefined end-point. Higher peak-exercise BNP level was associated with higher occurrence of adverse events $(p<0.0001)$. In multivariate analysis, second and third tertiles of peak-exercise BNP (T2: HR=2.9; $p=0.002$ and T3: $H R=5.3 ; p<0.0001$, respectively) were powerful predictors of events compared with the first tertile. Further adjustment for resting BNP provided comparable results (T2: $H R=2.8 ; p=0.003$ and $T 3: H R=5.0$; $p<0.0001)$. This relationship persisted in both subsets of patients with low or high resting BNP.

Conclusions This study reports that peak-exercise BNP level provides significant incremental prognostic value beyond what is achieved by demographic and echocardiographic data, as well as resting BNP level.

\section{INTRODUCTION}

Aortic valve replacement (AVR) is the only efficient treatment for aortic stenosis (AS). However, the optimal timing of AVR in asymptomatic patients with severe AS remains a matter of debate. Both the American College of Cardiology/American Heart Association (ACC/AHA) and the European Society of Cardiology (ESC) guidelines recommend AVR for patients with severe AS who present with symptoms or LV systolic dysfunction (ie, LVEF $<50 \%$ ). ${ }^{1} \quad{ }^{2}$ Patients with AS are often older, have more comorbidities and are less physically active, which make the assessment of symptoms challenging and unreliable. ${ }^{3-5}$ Hence, this raises the concern that a large proportion of these patients with AS could be operated too late in the course of the disease, which could negatively impact on the outcome. ${ }^{6-8}$ Previous studies reported that exercise testing, exercise-stress echocardiography and resting plasma levels of B-type natriuretic peptide (BNP) can improve risk stratification of patients with asymptomatic AS. ${ }^{9-15}$ The BNP response to exercise has been shown to be associated with outcomes in various cardiovascular diseases. $^{16-19}$ We hypothesised that plasma level of BNP after exercise provided incremental prognostic value beyond what is achieved by demographic, echocardiographic and resting BNP data.

\section{METHODS}

\section{Patient population}

We prospectively enrolled 211 asymptomatic patients with moderate to severe AS (peak aortic jet velocity $>2.5 \mathrm{~m} / \mathrm{s}$ and aortic valve area (AVA) $<1.5 \mathrm{~cm}^{2}$ ) and preserved LVEF in two centres (Quebec, $\mathrm{n}=40$; and Liège, $\mathrm{n}=171$ ). Patients with the following criteria were excluded: (i) moderate to severe aortic regurgitation or mitral valve disease; (ii) pregnant or lactating women; (iii) abnormal exercise test as previously defined; ${ }^{12}$ and (iv) estimated glomerular filtration rate $<60 \mathrm{~mL} /$ min (Modification of the Diet in Renal Disease (MDRD) formula). The study was approved by the relevant Ethics Committees, and patients signed a written informed consent.

\section{Clinical data}

Clinical data included age, gender, height, weight, body surface area (BSA), systolic and diastolic blood pressures, documented diagnoses of hypertension, diabetes, hypercholesterolaemia, and history of smoking, as previously defined. ${ }^{19}$

\section{Exercise protocol}

A symptom-limited graded bicycle exercise test was performed in the semisupine position on a dedicated tilting exercise table, following previously published settings. ${ }^{12}$

\section{Doppler echocardiographic data}

Doppler echocardiographic data were obtained at rest and at peak-exercise (peak-ex). 
The Doppler echocardiographic indices of AS severity included peak aortic jet velocity, peak and mean transvalvular pressure gradients, and AVA indexed to BSA. Severe AS was defined according to ACC/AHA-ESC guidelines. ${ }^{1}{ }^{2}$

The relative wall thickness and LV mass indexed to BSA were calculated. $^{20}$ LVEF was measured with the use of biplane Simpson method.

Left atrial (LA) area was obtained by planimetry at end systole from apical four-chamber view and was indexed to BSA. E to $\mathrm{e}^{\prime}$ ratio was calculated from the E-wave of mitral inflow and the average of septal and lateral mitral annulus $\mathrm{e}^{\prime}$-wave. Systolic pulmonary arterial pressure (SPAP) was evaluated from the regurgitant jet of the tricuspid insufficiency. ${ }^{12}$ Resting and exercise pulmonary hypertension (PHT) were defined as SPAP >50 and > $>00 \mathrm{mmHg}$, respectively. As a measure of global LV haemodynamic load, we calculated the valvulo-arterial impedance $\left(\mathrm{Z}_{\mathrm{va}}\right) .{ }^{21}$

\section{Plasma BNP level}

Venous blood samples for BNP measurements were drawn before echocardiography after $20 \mathrm{~min}$ of supine rest (resting $\mathrm{BNP}$ ) and at peak-ex, that is, within the 3 min following the end of exercise (peak-ex BNP) as previously validated. ${ }^{19}$

\section{Study end-point}

The study end-point was the occurrence of death or AVR motivated by development of symptoms or LV dysfunction (ie, class I indication). To ensure blinding, both resting and peak-ex BNP levels were not transmitted to the treating physician or surgeon.

\section{Statistical analysis}

Patients were divided into three groups according to tertiles of peak-ex BNP level. Continuous data were expressed as mean \pm SD and were tested for normality of distribution and homogeneity of variances with the Shapiro-Wilk and Levene tests, respectively. Groups were compared with one-way Analysis of Variance (ANOVA) followed by a Tukey's post hoc test when appropriate. Categorical data were expressed as percentage and groups were compared with the $\chi^{2}$ or Fischer's exact tests when appropriate. BNP levels were reported as median (IQR) and the natural logarithm transformation was applied to BNP levels. Difference between resting and peak-ex BNP levels was obtained with paired $t$ test and correlations between resting BNP and peak-ex BNP or absolute change in BNP ( $\delta$ BNP) were determined using Pearson's product-moment correlations. Multivariate linear regression analysis, including age, gender, peak-ex workload and exercise-induced increase in mean gradient, stroke volume index and $Z_{v a}$, was performed to identify the independent determinants of peak-ex BNP level. Kaplan-Meier curves and log-rank tests of the time-to-event data were used to assess the effect of tertiles of peak-ex BNP and of $\delta$ BNP on the composite end-point of death or AVR. The relationships among resting, peak-ex, $\delta$ BNP levels and event-free survival were assessed with the use of individual and multivariate Cox proportional-hazard analyses. The traditional risk factors of the composite of death or AVR and all variables with $\mathrm{p}$ value $<0.10$ in individual Cox analysis (ie, age, gender, resting mean gradient, resting $Z_{\mathrm{va}}$, resting indexed LA area and exercise-induced increase in heart rate, mean gradient and $Z_{\mathrm{va}}$ ) were entered in the multivariate Cox model. The proportional-hazards assumption was checked with the use of Schoenfeld residuals. The incremental predictive

Table 1 Clinical characteristics and exercise capacity data according to tertiles of peak-ex BNP level

\begin{tabular}{|c|c|c|c|c|c|}
\hline & $\begin{array}{l}\text { Whole Cohort } \\
n=211\end{array}$ & $\begin{array}{l}\text { Tertile } 1(6-42 \mathrm{pg} / \mathrm{mL}) \\
\mathrm{n}=71\end{array}$ & $\begin{array}{l}\text { Tertile } 2(43-91 \mathrm{pg} / \mathrm{mL}) \\
\mathrm{n}=70\end{array}$ & $\begin{array}{l}\text { Tertile } 3(92-956 \mathrm{pg} / \mathrm{mL}) \\
\mathrm{n}=70\end{array}$ & p Valuet \\
\hline \multicolumn{6}{|l|}{ Clinical data } \\
\hline Age, years & $68 \pm 11$ & $65 \pm 12$ & $69 \pm 10^{*}$ & $69 \pm 10^{*}$ & 0.03 \\
\hline Male gender, \% & $64 \%$ & $63 \%$ & $66 \%$ & $63 \%$ & 0.91 \\
\hline Height, cm & $167 \pm 8$ & $167 \pm 7$ & $168 \pm 10$ & $166 \pm 8$ & 0.50 \\
\hline Weight, kg & $74 \pm 13$ & $76 \pm 12$ & $76 \pm 12$ & $72 \pm 13$ & 0.21 \\
\hline Body surface area, $\mathrm{m}^{2}$ & $1.82 \pm 0.18$ & $1.82 \pm 0.18$ & $1.83 \pm 0.18$ & $1.81 \pm 0.19$ & 0.71 \\
\hline Body mass index, $\mathrm{kg} / \mathrm{m}^{2}$ & $26.6 \pm 3.7$ & $27.1 \pm 3.9$ & $26.8 \pm 3.5$ & $26.0 \pm 3.6$ & 0.27 \\
\hline Heart rate, beats/min & $70 \pm 12$ & $71 \pm 12$ & $70 \pm 10$ & $70 \pm 13$ & 0.99 \\
\hline History of hypertension, \% & $53 \%$ & $57 \%$ & $49 \%$ & $53 \%$ & 0.65 \\
\hline Systolic blood pressure, $\mathrm{mm} \mathrm{Hg}$ & $141 \pm 21$ & $142 \pm 24$ & $139 \pm 17$ & $142 \pm 21$ & 0.71 \\
\hline Diastolic blood pressure, $\mathrm{mm} \mathrm{Hg}$ & $77 \pm 11$ & $79 \pm 11$ & $74 \pm 10^{*}$ & $77 \pm 11$ & 0.04 \\
\hline Hypercholesterolaemia, \% & $46 \%$ & $48 \%$ & $43 \%$ & $47 \%$ & 0.83 \\
\hline Diabetes, \% & $14 \%$ & $13 \%$ & $12 \%$ & $17 \%$ & 0.44 \\
\hline History of smoking, \% & $27 \%$ & $24 \%$ & $31 \%$ & $28 \%$ & 0.65 \\
\hline Resting BNP level, pg/mL & $43(24-81)$ & $16(9-26)$ & $45(39-60)$ & $101(76-133)$ & By design \\
\hline \multicolumn{6}{|l|}{ Exercise testing data } \\
\hline Duration, min & $9.1 \pm 3.1$ & $9.0 \pm 2.6$ & $8.9 \pm 2.9$ & $9.4 \pm 3.6$ & 0.69 \\
\hline Peak-ex workload, watts & $99 \pm 35$ & $102 \pm 38$ & $101 \pm 35$ & $94 \pm 33$ & 0.35 \\
\hline Peak-ex heart rate, beats/min & $120 \pm 19$ & $124 \pm 20$ & $119 \pm 16$ & $118 \pm 19$ & 0.13 \\
\hline $\begin{array}{l}\text { Percent of predicted maximal } \\
\text { heart rate, \% }\end{array}$ & $91 \pm 12$ & $92 \pm 12$ & $91 \pm 11$ & $90 \pm 14$ & 0.83 \\
\hline Peak-ex systolic blood pressure, $\mathrm{mm} \mathrm{Hg}$ & $179 \pm 23$ & $184 \pm 23$ & $177 \pm 24$ & $177 \pm 22$ & 0.17 \\
\hline Peak-ex diastolic blood pressure, $\mathrm{mm} \mathrm{Hg}$ & $87 \pm 14$ & $90 \pm 14$ & $85 \pm 14$ & $86 \pm 13$ & 0.09 \\
\hline Peak-ex BNP level, pg/mL & $58(29-115)$ & $20(13-30)$ & $59(53-74)$ & $146(115-201)$ & By design \\
\hline
\end{tabular}


Table 2 Resting and peak-exercise echocardiographic data according to tertiles of peak-exercise BNP level

\begin{tabular}{|c|c|c|c|c|c|}
\hline & $\begin{array}{l}\text { Whole cohort } \\
n=211\end{array}$ & $\begin{array}{l}\text { Tertile } 1(6-42 \mathrm{pg} / \mathrm{mL}) \\
\mathrm{n}=71\end{array}$ & $\begin{array}{l}\text { Tertile } 2(43-91 \mathrm{pg} / \mathrm{mL}) \\
\mathrm{n}=70\end{array}$ & $\begin{array}{l}\text { Tertile } 3(92-956 \mathrm{pg} / \mathrm{mL}) \\
\mathrm{n}=70\end{array}$ & $\begin{array}{l}p \\
\text { Valuet }\end{array}$ \\
\hline \multicolumn{6}{|l|}{ Resting echocardiographic data } \\
\hline Stroke volume, mL & $84 \pm 19$ & $86 \pm 20$ & $86 \pm 19$ & $79 \pm 19$ & 0.05 \\
\hline Stroke volume index, $\mathrm{mL} / \mathrm{m}^{2}$ & $46 \pm 11$ & $48 \pm 11$ & $47 \pm 11$ & $44 \pm 11$ & 0.13 \\
\hline Peak aortic jet velocity, m/s & $4.0 \pm 0.7$ & $3.7 \pm 0.7$ & $4.1 \pm 0.7^{*}$ & $4.1 \pm 0.8^{*}$ & 0.001 \\
\hline $\begin{array}{l}\text { Peak transvalvular gradient, } \\
\mathrm{mm} \mathrm{Hg}\end{array}$ & $66 \pm 24$ & $59 \pm 21$ & $69 \pm 23^{*}$ & $71 \pm 25^{*}$ & 0.006 \\
\hline $\begin{array}{l}\text { Mean transvalvular gradient, } \\
\mathrm{mm} \mathrm{Hg}\end{array}$ & $41 \pm 15$ & $37 \pm 13$ & $43 \pm 15^{*}$ & $43 \pm 16^{*}$ & 0.02 \\
\hline Aortic valve area, $\mathrm{cm}^{2}$ & $0.93 \pm 0.21$ & $0.98 \pm 0.19$ & $0.90 \pm 0.20^{*}$ & $0.90 \pm 0.24^{*}$ & 0.03 \\
\hline Indexed aortic valve area, $\mathrm{cm}^{2} / \mathrm{m}^{2}$ & $0.51 \pm 0.12$ & $0.54 \pm 0.11$ & $0.49 \pm 0.10^{*}$ & $0.50 \pm 0.13^{*}$ & 0.03 \\
\hline Indexed left atrial area, $\mathrm{cm}^{2} / \mathrm{m}^{2}$ & $11.6 \pm 3.3$ & $11.0 \pm 3.0$ & $10.9 \pm 3.1$ & $12.8 \pm 3.4^{*} \ddagger$ & 0.0007 \\
\hline E to $e^{\prime}$ & $10.8 \pm 4.2$ & $9.9 \pm 3.9$ & $11.2 \pm 3.8$ & $11.4 \pm 4.8$ & 0.15 \\
\hline Pulmonary hypertension, \% & $3 \%$ & $1 \%$ & $1 \%$ & $7 \%$ & 0.09 \\
\hline Relative wall thickness & $0.51 \pm 0.12$ & $0.48 \pm 011$ & $0.51 \pm 0.09$ & $0.54 \pm 0.15^{*}$ & 0.04 \\
\hline LV mass index, $\mathrm{g} / \mathrm{m}^{2}$ & $124 \pm 45$ & $123 \pm 47$ & $126 \pm 47$ & $123 \pm 41$ & 0.88 \\
\hline LVEF, \% & $66 \pm 7$ & $67 \pm 8$ & $66 \pm 6$ & $66 \pm 8$ & 0.66 \\
\hline $\begin{array}{l}\text { Valvulo-arterial imp., mm Hg/ } \\
\mathrm{mL} \mathrm{m} \mathrm{m}^{2}\end{array}$ & $4.1 \pm 1.1$ & $3.9 \pm 0.8$ & $4.0 \pm 1.0$ & $4.5 \pm 1.3^{*} \ddagger$ & 0.003 \\
\hline \multicolumn{6}{|c|}{ Peak-exercise-stress echocardiographic data } \\
\hline Stroke volume, $\mathrm{mL}$ & $94 \pm 29$ & $91 \pm 27$ & $94 \pm 32$ & $97 \pm 30$ & 0.49 \\
\hline Stroke volume index, $\mathrm{mL} / \mathrm{m}^{2}$ & $49 \pm 16$ & $47 \pm 15$ & $49 \pm 17$ & $50 \pm 16$ & 0.49 \\
\hline Peak aortic jet velocity, m/s & $4.6 \pm 0.8$ & $4.3 \pm 0.7$ & $4.7 \pm 0.7^{*}$ & $4.7 \pm 0.8^{*}$ & 0.01 \\
\hline $\begin{array}{l}\text { Peak transvalvular gradient, } \\
\mathrm{mm} \mathrm{Hg}\end{array}$ & $86 \pm 28$ & $78 \pm 27$ & $89 \pm 26^{*}$ & $90 \pm 30^{*}$ & 0.02 \\
\hline $\begin{array}{l}\text { Mean transvalvular gradient, } \\
\mathrm{mm} \mathrm{Hg}\end{array}$ & $53 \pm 19$ & $48 \pm 16$ & $56 \pm 18^{*}$ & $56 \pm 21^{*}$ & 0.01 \\
\hline Aortic valve area, $\mathrm{cm}^{2}$ & $1.01 \pm 0.29$ & $1.09 \pm 0.26$ & $0.97 \pm 0.29^{*}$ & $0.98 \pm 0.30$ & 0.03 \\
\hline Indexed aortic valve area, $\mathrm{cm}^{2} / \mathrm{m}^{2}$ & $0.56 \pm 0.16$ & $0.60 \pm 0.15$ & $0.53 \pm 0.16^{*}$ & $0.55 \pm 0.17$ & 0.03 \\
\hline Pulmonary hypertension, $\%$ & $32 \%$ & $23 \%$ & $34 \%$ & $39 \%$ & 0.10 \\
\hline LVEF, \% & $68 \pm 9$ & $68 \pm 10$ & $69 \pm 8$ & $67 \pm 9$ & 0.42 \\
\hline $\begin{array}{l}\text { Valvulo-arterial imp., } \mathrm{mm} \mathrm{Hg} / \\
\mathrm{mL} \mathrm{m}^{2}\end{array}$ & $5.1 \pm 1.9$ & $5.2 \pm 1.8$ & $5.2 \pm 1.9$ & $5.0 \pm 1.9$ & 0.82 \\
\hline
\end{tabular}

Values are mean \pm SD.

tp Value of the one-way ANOVA. The following symbols indicate the significance of the Tukey's post hoc test: * $p<0.05$ from 'Tertile 1'; $\neq p<0.05$ from 'Tertile 2'.

BNP, B-type natriuretic peptide.

value of peak-ex BNP was assessed by calculating the net reclassification improvement (NRI) for 1-year events (death or AVR) using the NRI \& IDI program codes downloaded online (http://www.ucr. uu.se/en/index.php/ucr-statistics/program-code/

306-nri-and-idi). ${ }^{22}$ Category cut-points were defined as observed event rate at 1-year (ie, $28 \%$ ) or tertiles of risk probabilities determined using the model with established risk factors (ie, $<19 \%$, $19 \%-35 \%$, and $\geq 35 \%$ ). Patients were reclassified according to 1-year predicted probabilities after addition of peak-ex BNP. The receiver operating characteristic (ROC) curve analyses were performed to determine the accuracy (area under the curve, AUC) as well as the best cut-point values for resting and peak-ex BNP levels to predict 1-year events expressed as a binary outcome. Statistical analyses were done with Stata Software (V.11.0). A p value $<0.05$ was considered statistically significant.

\section{RESULTS}

\section{Population characteristics}

Baseline characteristics of the population are depicted in tables 1 and 2. Severe AS was present in 157 (74\%) patients. The baseline characteristics (all $\mathrm{p}>0.05$; data not shown) and 1-year event rates $(65 \%$ vs $73 \% ; p=0.87)$ were similar in the subset of patients enrolled from Canada versus those enrolled from Belgium.

\section{Response of BNP to exercise}

BNP level increased significantly from rest to peak-ex $(\mathrm{p}<0.0001$; figure 1A) and both resting and peak-ex BNP levels were strongly correlated $(r=0.89, p<0.0001$; figure $1 B)$. The exercise-induced absolute change in BNP was $9(2-29) \mathrm{pg} / \mathrm{mL}$ and $182(86 \%)$ patients exhibited an increase in BNP during exercise (ie, $\delta \mathrm{BNP}>0$ ). There was a significant but modest correlation between resting BNP and absolute $\delta$ BNP $(r=0.31$, $\mathrm{p}<0.0001$; figure 1C). Peak-ex BNP correlated weakly and negatively with peak-ex workload $(\mathrm{r}=-0.16 ; \mathrm{p}=0.03)$.

Patients in the second (T2) and third (T3) tertiles of peak-ex $\mathrm{BNP}$ had higher resting BNP level $(\mathrm{p}<0.0001$, table 1$)$; they were significantly older $(p=0.03$, table 1$)$ and had more severe AS (table 2) compared with patients in the first tertile (T1). The exercise testing data were similar between the three tertiles (table 1). Resting indexed LA area and $Z_{v a}$ were higher in T3 compared with T1 and T2 (table 2).

\section{Correlates of peak-ex BNP}

Resting Doppler echocardiographic variables associated with peak-ex BNP level were mean gradient, indexed LA area and $Z_{\mathrm{va}}$ (table 3). After adjustment for age, gender and peak-ex workload, as well as for exercise-stress echocardiographic variables associated with peak-ex BNP level (table 3, Multivariate 

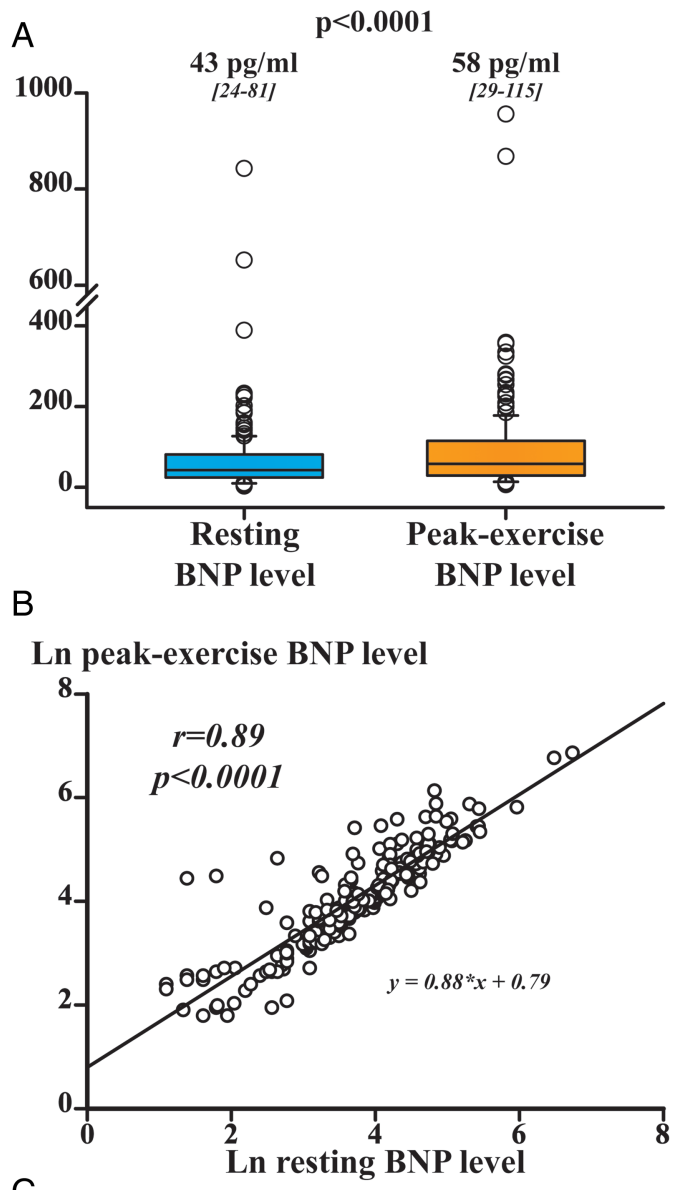

C

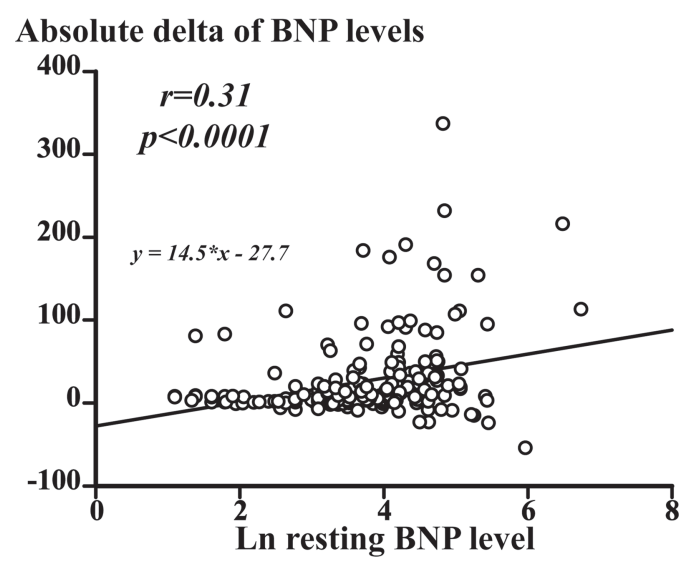

Figure 1 Distribution and association among resting, peak-exercise and $\delta$ B-type natriuretic peptide (BNP) levels in the whole cohort $(n=211)$. (A) Shows comparison between resting and peak-exercise BNP levels; the box shows 25th and 75th percentiles, the median line on the box shows the median value, and error bars the 10th and 90th percentiles; circles are outliers; the numbers of the top of the graph are median (IQR) value of BNP levels. (B) Shows correlation between In resting BNP and In peak-exercise BNP level. (C) Shows correlation between In resting BNP and absolute $\delta$ of BNP levels; $r$ and $p$ value are from Pearson's product-moment correlations; several data point were superimposed; the formula represents the equation of the regression line.

Model), resting mean gradient $(\mathrm{p}=0.03)$, indexed LA area $(p=0.04)$ and $Z_{v a}(p=0.009)$ remained the only variables associated with peak-ex BNP level.
Table 3 Correlates of peak-exercise BNP level in the whole cohort $(\mathrm{n}=211)$

\begin{tabular}{|c|c|c|c|c|}
\hline \multirow[b]{2}{*}{ Ln peak-exercise BNP } & \multicolumn{2}{|l|}{ Individual } & \multicolumn{2}{|c|}{ Multivariate model* } \\
\hline & $\beta$ coeff. \pm SE & $p$ Value & $\beta$ coeff. \pm SE & p Value \\
\hline $\begin{array}{l}\text { Resting mean transvalvular } \\
\text { gradient, } \mathrm{mm} \mathrm{Hg}\end{array}$ & $0.25 \pm 0.004$ & 0.0003 & $0.16 \pm 0.005$ & 0.03 \\
\hline $\begin{array}{l}\text { Resting indexed left atrial area, } \\
\mathrm{cm}^{2} / \mathrm{m}^{2}\end{array}$ & $0.23 \pm 0.02$ & 0.001 & $0.15 \pm 0.02$ & 0.04 \\
\hline $\begin{array}{l}\text { Resting valvulo-arterial } \\
\text { impedance, } \mathrm{mm} \mathrm{Hg} / \mathrm{mL} \mathrm{m}^{2}\end{array}$ & $0.25 \pm 0.06$ & 0.0002 & $0.20 \pm 0.07$ & 0.009 \\
\hline
\end{tabular}

\section{Association between resting and peak-ex BNP levels} and outcome

During a mean follow-up of $1.8 \pm 1.3$ years, 104 prespecified events occurred (ie, seven deaths and 97 AVRs) in the whole cohort. Given that, from a clinical standpoint, the most challenging subset of patients is the one with asymptomatic severe AS, our analyses of outcomes were focused on this subset and the results for the whole cohort are presented in online supplementary table S1 and online supplementary figures S1 and S2. In the subset with severe AS $(n=157$ (74\%); mean follow-up: 1.5 \pm 1.2 years), 87 prespecified events occurred (ie, seven deaths and 78 AVRs) resulting in a cardiac event-free survival of 72 $\pm 4 \%, 48 \pm 5 \%$ and $39 \pm 5 \%$ at 1,2 and 3 years, respectively.

When using BNP variables in continuous format, both resting and peak-ex BNP levels were associated with a 1.5-fold increase in risk of events (for each $100 \mathrm{pg} / \mathrm{mL}$ increase) $(\mathrm{p}<0.0001$; table 4). After adjustment for clinical, resting and exercise-stress echocardiographic data, resting and peak-ex BNP levels were associated with a 1.4 -fold $(p=0.007)$ and 1.4 -fold $(p<0.0001)$ increase in the risk of events, respectively (table 4, Model \#1). The inclusion of $\delta$ BNP in place of peak-ex BNP also provided similar results $(\mathrm{HR}=3.4 ; \mathrm{p}<0.0001$; table 4, Model \#2).

There was a significant graded relationship between tertiles of peak-ex BNP level and occurrence of events $(\mathrm{p}<0.0001$; figure $2 \mathrm{~A}$; table 4). The analysis of tertiles of $\delta$ BNP provided similar results as tertiles of peak-ex BNP ( $p<0.0001$; figure $2 \mathrm{~B}$; table 4$)$. After multivariate adjustment (table 4, Model \#1), T2 and T3 of peak-ex BNP remained associated with significant increase in risk of events. Further adjustment for resting BNP level provided similar results: tertiles of peak-ex BNP levels were associated with increased risk of events, whereas resting BNP was not (table 4, Model \#2). Additional adjustment for exercise PHT or exercise workload did not change the results. The multivariate analysis including resting BNP expressed in tertiles and peak-ex BNP in continuous variable was consistent with results presented above (see online supplementary table S2). Furthermore, when we included both resting and peak-ex BNPs in continuous format into the multivariate model, peak-ex BNP remained significantly associated with higher risk of events $(\mathrm{HR}=3.4 ; \mathrm{p}<0.0001)$, whereas resting $\mathrm{BNP}$ did not.

\section{Incremental prognostic value of peak-ex BNP}

The analysis of the effect of peak-ex BNP according to median value of resting $\mathrm{BNP}$ (ie, resting $\mathrm{BNP}$ level $>$ or $\leq 48 \mathrm{pg} / \mathrm{mL}$ ) showed that high peak-ex BNP, dichotomised to median value in both subsets of resting BNP level (ie, $>$ or $\leq 38 \mathrm{pg} / \mathrm{mL}$ in patients with low resting BNP, and $>$ or $\leq 120 \mathrm{pg} / \mathrm{mL}$ in patients with high resting $\mathrm{BNP}$ ), was associated with significantly higher 
Table 4 Impact of BNP level on event-free survival in patients with severe AS ( $n=157)$

\begin{tabular}{|c|c|c|c|c|c|c|}
\hline & \multicolumn{2}{|l|}{ Individual } & \multicolumn{2}{|c|}{ Multivariate models \#1 } & \multicolumn{2}{|c|}{ Multivariate models \#2 } \\
\hline & HR $(95 \% \mathrm{Cl})$ & p Value & HR $(95 \% \mathrm{Cl})$ & $\mathrm{p}$ Value & HR $(95 \% \mathrm{Cl})$ & $\mathrm{p}$ Value \\
\hline \multicolumn{7}{|l|}{ Resting BNP level } \\
\hline Resting BNP level (per 100 pg/mL increase) & $1.5(1.2$ to 1.8$)$ & $<0.0001$ & $1.4(1.1$ to 1.8$)$ & 0.007 & - & - \\
\hline \multicolumn{7}{|l|}{ Peak-exercise BNP level } \\
\hline Peak-exercise BNP level (per 100 pg/mL increase) & $1.5(1.4$ to 1.7$)$ & $<0.0001$ & $1.4(1.2$ to 1.7$)$ & $<0.0001$ & - & - \\
\hline \multicolumn{7}{|l|}{ Tertiles of peak-exercise BNP level ${ }^{*}$} \\
\hline Tertile 1 (6 to $46 \mathrm{pg} / \mathrm{mL}$ ) & 1.0 (referent) & - & 1.0 (referent) & - & 1.0 (referent) & - \\
\hline Tertile 2 (47 to $94 \mathrm{pg} / \mathrm{mL}$ ) & $2.6(1.4$ to 5.0$)$ & 0.0003 & $2.9(1.5$ to 5.7$)$ & 0.002 & $2.8(1.4$ to 5.7$)$ & 0.003 \\
\hline Tertile 3 (95 to $956 \mathrm{pg} / \mathrm{mL}$ ) & $6.1(3.2$ to 11.4$)$ & $<0.0001$ & $5.3(2.6$ to 10.8$)$ & $<0.0001$ & $5.0(2.3$ to 11.0$)$ & $<0.0001$ \\
\hline Resting BNP level (per 100 pg/mL increase) & $1.5(1.2$ to 1.8$)$ & $<0.0001$ & - & - & $1.1(0.7$ to 1.5$)$ & 0.75 \\
\hline \multicolumn{7}{|l|}{$\delta$ Of BNP levels } \\
\hline Absolute difference of BNP levels (per $100 \mathrm{pg} / \mathrm{mL}$ increase from rest) & $3.6(2.6$ to 4.9$)$ & $<0.0001$ & - & - & $3.4(2.2$ to 5.3$)$ & $<0.0001$ \\
\hline Resting BNP level (per $100 \mathrm{pg} / \mathrm{mL}$ increase) & $1.5(1.2$ to 1.8$)$ & $<0.0001$ & - & - & $1.0(0.7$ to 1.3$)$ & 0.95 \\
\hline \multicolumn{7}{|l|}{ Tertiles of $\delta$ BNP levels } \\
\hline Tertile 1 (-54 to 4$)$ & 1.0 (referent) & - & 1.0 (referent) & - & 1.0 (referent) & - \\
\hline Tertile 2 (4 to 20 ) & $2.5(1.3$ to 5.7$)$ & 0.0006 & $3.1(1.4$ to 6.9$)$ & 0.006 & $3.0(1.2$ to 6.2$)$ & 0.008 \\
\hline Tertile 3 (20 to 337) & $8.4(4.1$ to 17.1$)$ & $<0.0001$ & $7.7(3.4$ to 17.4$)$ & $<0.0001$ & 7.1 (3.1 to 16.1$)$ & $<0.0001$ \\
\hline Resting BNP level (per $100 \mathrm{pg} / \mathrm{mL}$ increase) & $1.5(1.2$ to 1.8$)$ & $<0.0001$ & - & - & $1.2(0.9$ to 1.6$)$ & 0.12 \\
\hline
\end{tabular}

rate of events in both subsets of resting BNP level $(p<0.0001$; figure 3). Among patients with resting BNP level $\leq 48 \mathrm{pg} / \mathrm{mL}$, peak-ex BNP $>38 \mathrm{pg} / \mathrm{mL}$ was associated with significantly higher risk of events $(\mathrm{HR}=3.1,95 \% \mathrm{CI} 1.2$ to $7.0, \mathrm{p}=0.02)$ after adjustment for age, gender, mean gradient, indexed LA area, and exercise-induced increase in heart rate and mean gradient.

The addition of peak-ex BNP level in the multivariate model including resting BNP (Model \#2, table 4) significantly improved the predictive value of the model: the two-category NRI for 1-year events was 0.17 (95\% CI 0.02 to $0.33 ; \mathrm{p}=0.03$ ) and the three-category NRI was 0.25 (95\% CI 0.04 to 0.46 ; $\mathrm{p}=0.02$ ) (see online supplementary tables S3 and S4). Furthermore, in the ROC curve analyses, the AUC to predict 1 -year events was higher with peak-ex BNP compared with resting BNP (73 \pm 5 vs $61 \pm 5 \% ; \mathrm{p}<0.0001)$. The best cut-point value was $86 \mathrm{pg} / \mathrm{mL}$ for peak-ex BNP and $58 \mathrm{pg} / \mathrm{mL}$ for resting BNP. In multivariate Cox analysis, peak-ex BNP level $>86 \mathrm{pg} /$ $\mathrm{mL}$ was significantly associated with increased risk of events $(\mathrm{HR}=3.5 ; \mathrm{p}=0.01)$ whereas resting $\mathrm{BNP}>58 \mathrm{pg} / \mathrm{mL}$ was not $(\mathrm{HR}=1.1 ; \mathrm{p}=0.86)$ (see online supplementary table $\mathrm{S} 5)$.

\section{DISCUSSION}

The main findings of this study are: (1) Peak-ex BNP is a powerful predictor of outcome and provides important incremental prognostic value beyond what is achieved by demographic, and echocardiographic data, as well as resting BNP level. (2) This relationship remains significant in the subsets of patients with low resting BNP.

\section{BNP response to exercise}

Resting blood level of BNP is increased in various cardiac diseases as compared with healthy subjects and is associated with higher LV pressure or volume overload. ${ }^{24}$ In patients with AS, resting BNP level is useful to enhance risk stratification in AS patients. ${ }^{13-15} 2425$ In response to exercise, BNP level may increase both in normal healthy subjects ${ }^{26}$ and patients with cardiovascular diseases. ${ }^{16-19}$ In the present study of asymptomatic AS patients, we showed a significant increase in BNP during exercise. The overall correlation between resting and peak-ex BNP levels was strong but with important inter-individual variability in the BNP response to exercise; that is, some patients with low resting level of BNP had marked increase in BNP during exercise, whereas others had no significant change. Large increase in BNP during exercise may be a surrogate marker for the adverse repercussions of the haemodynamic burden as well as of the concomitant diseases on the cardiac chambers and may therefore help to identify patients who are at a more advanced stage of the disease at the valvular, arterial and/or ventricular levels. This hypothesis is supported by the independent association we found between peak-ex BNP level and (i) resting AS severity, (ii) global (valvulo-arterial) LV haemodynamic burden and (iii) LA enlargement.

\section{Peak-ex BNP and outcome}

In this large bi-centric prospective series of patients, peak-ex BNP level was significantly associated with higher risk of events, even after adjustment for clinical, resting and peak-ex echocardiographic data and resting BNP level. The results of multivariate analyses clearly demonstrated that peak-ex BNP provides incremental prognostic value beyond clinical and resting variables. Furthermore, this incremental value was also observed in the subset of patients with low BNP at rest. Hence, peak-ex BNP allows the cardiologist to re-stratify patients who would be identified as low risk otherwise.

\section{Clinical implications}

The present findings suggest that measurement of BNP during exercise testing or exercise-stress echocardiography may be useful to enhance risk stratification and thus the clinical management of asymptomatic patients with severe AS. Patients exhibiting a marked increase in BNP during exercise are at much higher risk for adverse events in the short-term and thus require 
A

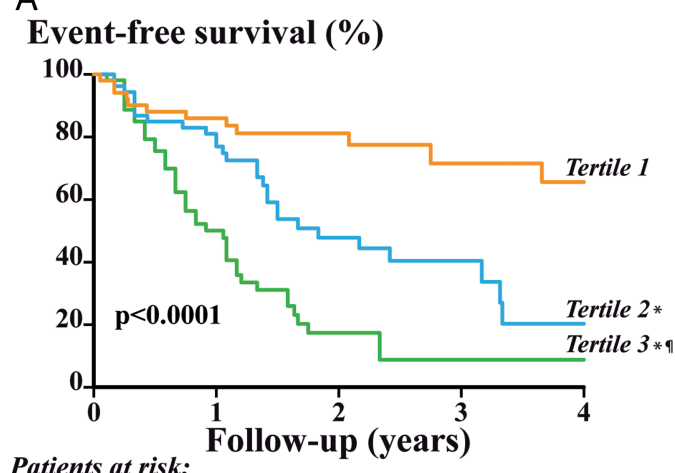

Patients at risk:

$\begin{array}{lcccc}51 & 40 & 22 & 12 & 10- \\ 53 & 40 & 14 & 7 & 3 \\ 53 & 22 & 5 & 1 & 1\end{array}$

B

Event-free survival (\%)

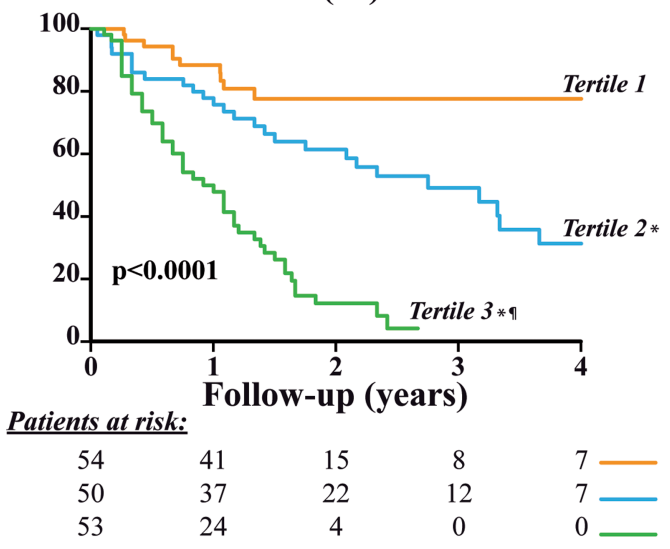

Figure 2 Comparison of the event-free survival curves for tertiles of peak-exercise B-type natriuretic peptide (BNP) level and tertiles of $\delta$ BNP levels in patients with severe aortic stenosis (AS) $(n=157)$. This figure shows the event-free survival curves for the composite end-point of death or aortic valve replacement in patients with severe AS $(n=157)$ according to tertiles of peak-exercise BNP level $(A)$ and tertiles of $\delta$ BNP levels (B). The symbols indicate the significant differences between groups: * $p<0.05$ versus 'Tertile 1'; $9 p<0.05$ versus 'Tertile 2 '. The numbers at the bottom of the graph represent the number of patients at risk at each follow-up year. The $p$ value is that of the log-rank test.

more frequent and closer clinical and echocardiographic follow-up.

However, BNP levels measured at rest or during exercise are not specific to AS severity alone but rather reflect the total burden of disease(s) on the LV and other cardiac chambers. They should thus be interpreted in light of the parameters more specifically reflecting AS severity as well as arterial and global loads.

It is often technically difficult to obtain valid exercise haemodynamic data in all patients, and a circulating biomarker such as BNP could thus be highly valuable in this context. The measurement of BNP indeed has the advantage of being easy, fast and reproducible and it can be combined with exercise-stress echocardiography or with standard treadmill exercise test. Further studies are, however, needed to determine if the present findings obtained in the semisupine position can be transposed to the setting of upright exercise test.

\section{Strengths and limitations}

The primary end-point was defined as the composite of AVR and death and was, in large part, driven by the former event (87 AVRs and seven deaths in the severe AS subset). AVR may

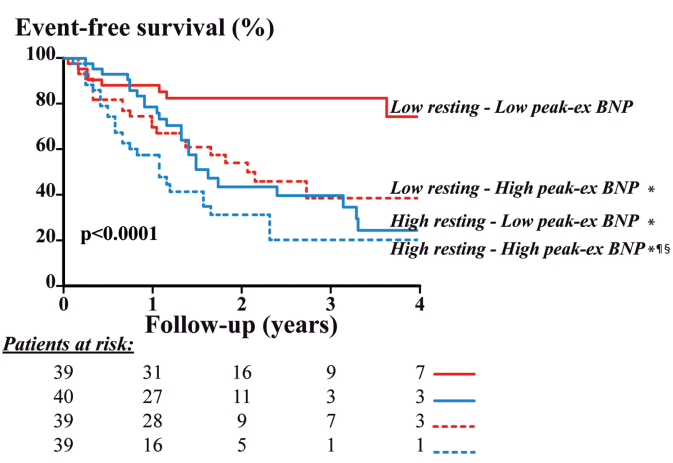

Figure 3 Comparison of the event-free survival curves for median peak-exercise B-type natriuretic peptide (BNP) level in the subsets of patients with high and low resting BNP level in patients with severe aortic stenosis $(n=157)$. This figure shows the event-free survival curves for the composite end-point of death or aortic valve replacement in the subsets of patients with low versus high resting BNP level (red vs blue curves, respectively), according to low versus high peak-exercise BNP (full vs dotted lines, respectively). The symbols indicate the significant difference between groups: * $p<0.05$ versus 'Low resting-Low peak-exercise BNP level'; I $p<0.05$ versus 'Low resting-High peak-exercise BNP level'; $\$ p<0.05$ versus 'High resting-Low peak-exercise BNP level'. The numbers at the bottom of the graph represent the number of patients at risk at each follow-up year. The $p$ value is that of the log-rank test.

be influenced by physician's perception and interpretation of disease severity and patient's symptomatic status. However, this study included only patients who were 'truly' asymptomatic at baseline (ie, with normal exercise test). The treating physicians were blinded to BNP data and only AVR motivated by the development of symptoms or LV systolic dysfunction was classified as event in the composite end-point. Furthermore, all multivariate models were adjusted for the baseline stenosis severity (ie, mean gradient at rest and during exercise) and peak-ex BNP remained an independent predictor of events despite this adjustment.

\section{Key messages}

What is known on this subject?

Some studies have reported that dosage of resting plasma B-type natriuretic peptide (BNP), exercise testing and exercise-stress echocardiography may be useful to predict outcomes and therefore improve risk stratification of patients with asymptomatic aortic stenosis (AS).

\section{What might this study add?}

The results of this study suggest that BNP measured at peak-exercise provides significant incremental prognostic value beyond what is achieved by demographic data, resting and exercise echocardiographic data, as well as resting BNP level. These findings provide strong impetus for systematic measurement of BNP during exercise test in the AS population.

\section{How might this impact on clinical practice?}

The findings of this study suggest that asymptomatic patients with severe AS who display a marked increase in BNP during exercise could be at higher risk of events. Measurement of BNP during exercise may thus be useful to enhance risk stratification and adjust the timing of clinical and echocardiographic follow-up in these patients. 
There were not enough patients to perform a separate analysis of mortality.

Due to the variability of the cut-off values of BNP reported in the literature to predict outcomes, we elected to use continuous variables or tertiles of BNP. Given the graded increase in the risk of adverse events reported in the present study, it would be misleading to propose a precise cut-point value for the prediction of outcome.

Acknowledgements We thank Isabelle Fortin, Jocelyn Beauchemin, Martine Parent and Carmine Cellentano for their help in data collection and management.

Contributors RC, JM, PL and PP designed the study and prepared the manuscript. $\mathrm{RC}$ performed data and statistical analyses. $\mathrm{RC}$ and JM wrote the first draft of the manuscript. RD and $\mathrm{ZH}$ participated in the recruitment of patients. PL and PP supervised the acquisition and analyses of echocardiographic images. All authors reviewed the manuscript and contributed to scientific content. PL and PP validated the final version and are responsible for the overall content.

Funding This work was supported, in part, by grant MOP-114997 from Canadian Institutes of Health Research (CIHR), Ottawa, Ontario, Canada, and a grant from the Foundation of the Quebec Heart and Lung Institute.

Competing interests RC was supported by a studentship grant of International Chair of Cardiometabolic Risk, Québec, Québec, Canada. JM is research associate from the F.R.S-FNRS, Brussels, Belgium, and received grants from the Fonds Léon Fredericq, Liège, Belgium. MA is a research scholar from the Fonds de recherche en santé du Québec (FRSQ), Montreal, Québec, Canada. PP holds the Canada Research Chair in Valvular Heart Diseases, CIHR.

Patient consent Obtained.

Ethics approval Ethics Committees of the Quebec Heart and Lung Institute and CHU Sart Tilman.

Provenance and peer review Not commissioned; externally peer reviewed.

\section{REFERENCES}

1 Vahanian A, Alfieri O, Andreotti F, et al. Guidelines on the management of valvular heart disease (version 2012). Joint task force on the management of valvular heart disease of the European Society of Cardiology (ESC); European Association for Cardio-Thoracic Surgery (EACTS). Eur Heart J 2012;33:2451-96.

2 Nishimura RA, Otto CM, Bonow RO, et al. 2014 AHA/ACC guideline for the management of patients with valvular heart disease. J Am Coll Cardiol 2014;63:2438-88.

3 Otto CM, Burwash IG, Legget ME, et al. Prospective study of asymptomatic valvular aortic stenosis. Clinical, echocardiographic, and exercise predictors of outcome. Circulation 1997;95:2262-70.

4 Malouf J, Le TT, Pellikka P, et al. Aortic valve stenosis in community medical practice: determinants of outcome and implications for aortic valve replacement. J Thorac Cardiovasc Surg 2012;144:1421-7.

5 Rosenhek R, Binder T, Porenta G, et al. Predictors of outcome in severe, asymptomatic aortic stenosis. N Engl J Med 2000;343:611-17.

6 Kang DH, Park SJ, Rim JH, et al. Early surgery versus conventional treatment in asymptomatic very severe aortic stenosis. Circulation 2010;121:1502-9.

7 Carabello BA. Aortic valve replacement should be operated on before symptom onset. Circulation 2012:126:112-17.
8 Shah PK. Severe aortic stenosis should not be operated on before symptom onset. Circulation 2012;126:118-25

9 Picano $E$, Pibarot $P$, Lancellotti $P$, et al. The emerging role of exercise testing and stress echocardiography in valvular heart disease. J Am Coll Cardio 2009:54:2251-60.

10 Lancellotti $\mathrm{P}$, Lebois $\mathrm{F}$, Simon $\mathrm{M}$, et al. Prognostic importance of quantitative exercise Doppler echocardiography in asymptomatic valvular aortic stenosis. Circulation 2005;112:1377-82.

11 Maréchaux S, Hachicha Z, Bellouin A, et al. Usefulness of exercise stress echocardiography for risk stratification of true asymptomatic patients with aortic valve stenosis. Eur Heart J 2010;31:1390-7.

12 Lancellotti $P$, Magne J, Donal $E$, et al. Determinants and prognostic significance of exercise pulmonary hypertension in asymptomatic severe aortic stenosis. Circulation 2012;126:851-9.

13 Bergler-Klein J, Klaar U, Heger M, et al. Natriuretic peptides predict symptom-free survival and postoperative outcome in severe aortic stenosis. Circulation 2004;109:2302-8

14 Lim P, Monin JL, Monchi M, et al. Predictors of outcome in patients with severe aortic stenosis and normal left ventricular function: role of B-type natriuretic peptide. Eur Heart J 2004;25:2048-53.

15 Lancellotti P, Moonen M, Magne J, et al. Prognostic effect of long-axis left ventricular dysfunction and B-type natriuretic peptide levels in asymptomatic aortic stenosis. Am J Cardiol 2010;105:383-8.

16 Foote RS, Pearlman JD, Siegel $\mathrm{AH}$, et al. Detection of exercise-induced ischemia by changes in B-type natriuretic peptides. J Am Coll Cardiol 2004

44:1980-7.

17 Bergeron S, Moller JE, Bailey KR, et al. Exertional changes in circulating cardiac natriuretic peptides in patients with suggested coronary artery disease. J Am Soc Echocardiogr 2006;19:772-6.

18 Kato M, Kinugawa T, Ogino K, et al. Augmented response in plasma brain natriuretic peptide to dynamic exercise in patients with left ventricular dysfunction and congestive heart failure. J Intern Med 2000;248:309-15.

19 Magne J, Mahjoub H, Pibarot $P$, et al. Prognostic importance of exercise brain natriuretic peptide in asymptomatic degenerative mitral regurgitation. Eur J Heart Fail 2012;14:1293-302

20 Lang RM, Bierig M, Devereux RB, et al. Recommendations for chamber quantification: a report from the American Society of Echocardiography's Guidelines and Standards Committee and the Chamber Quantification Writing Group, developed in conjunction with the European Association of Echocardiography, a branch of the European Society of Cardiology. J Am Soc Echocardiogr 2005:18:1440-63.

21 Hachicha Z, Dumesnil JG, Pibarot P. Usefulness of the valvuloarterial impedance to predict adverse outcome in asymptomatic aortic stenosis. J Am Coll Cardiol 2009;54:1003-11

22 Pencina MJ, D'Agostino RB Sr, D'Agostino RB Jr, et al. Evaluating the added predictive ability of a new marker: from area under the ROC curve to reclassification and beyond. Stat Med 2008;27:157-72.

23 Pencina MJ, D'Agostino RB Sr, Steyerberg EW. Extensions of net reclassification improvement calculations to measure usefulness of new biomarkers. Stat Med 2011:30:11-21.

24 Steadman CD, Ray S, Ng LL, et al. Natriuretic peptides in common valvular heart disease. J Am Coll Cardiol 2010:55:2034-48.

25 Gerber IL, Stewart RA, Legget ME, et al. Increased plasma natriuretic peptide levels reflect symptom onset in aortic stenosis. Circulation 2003:107:1884-90.

26 Huang WS, Lee MS, Perng HW, et al. Circulating brain natriuretic peptide values in healthy men before and after exercise. Metabolism 2002:51:1423-6. 